\title{
Visão das cores em escolares: avaliação de um novo teste
}

\author{
Color vision in school children: evaluation of a new test
}

\author{
Guilherme M. Martins ${ }^{1}$, Marcela F. Bordaberry ${ }^{1}$, Zélia M.S Corrêa ${ }^{1}$, Michel B. Mânica ${ }^{2}$, \\ Juliano C. Costa ${ }^{3}$, Nelson Telichevesky ${ }^{1}$, Ítalo M. Marcon ${ }^{1}$
}

\section{Resumo}

Objetivos: comparar os resultados de um teste de visão de cores padrão (teste de Ishihara) com um teste criado pelos autores (teste do giz de cera) na detecção da discromatopsia congênita.

Pacientes e Métodos: foi realizado um estudo transversal com 712 crianças da rede escolar pública (3 escolas) e privada (1 escola) de Porto Alegre, Rio Grande do Sul. Desta amostra aleatória, haviam sido excluídas crianças com dificuldades na alfabetização e doenças sistêmicas e oculares reconhecidas. Foram aplicados seqüencialmente os testes de Ishihara (versão resumida de 14 pranchas) e o teste do giz de cera (desenvolvido pelos autores). Cada teste foi aplicado por examinadores diferentes e avaliados por um terceiro examinador.

Resultados: o teste do giz de cera apresentou especificidade de $100 \%(99,3-100 \%)$ e sensibilidade de $38,5 \%$ (15,1-67,7\%), quando comparado ao teste de Ishihara. A prevalência da discromatopsia congênita nesta amostra populacional foi de 2,6\% nos meninos e $0,9 \%$ nas meninas.

Conclusões: o teste do giz de cera apresentou especificidade superior ao teste de Ishihara no grupo estudado, mas sua sensibilidade não se mostrou adequada para um teste de rastreamento. Há necessidade de aperfeiçoá-lo para aumentar sua sensibilidade, isto é, sua capacidade de detectar a discromatopsia congênita.

J Pediatr (Rio J) 2001; 77 (4): 327-30: teste da visão de cores, discromatopsia, teste de Ishihara, saúde escolar.

\section{Introdução}

A discromatopsia congênita ou daltonismo é uma deficiência que afeta a visão das cores ${ }^{1}$. A prevalência é de aproximadamente $7 \%$ nos homens e $0,5 \%$ nas mulheres. Esse predomínio do sexo masculino se deve ao fato desta patologia ser ligada ao cromossomo $\mathrm{X}^{2}$.

Muitas crianças pequenas confundem as cores, apesar de sua acuidade visual estar normal. Testar a visão das cores

1. Serviço de Oftalmologia da Santa Casa de Misericórdia de Porto Alegre, Porto Alegre, RS.

2. Acadêmico de Medicina da Fundação Faculdade Federal de Ciências Médicas de Porto Alegre, Porto Alegre, RS.

3. Acadêmico de Medicina da Universidade Luterana do Brasil (ULBRA), Canoas, RS.

\begin{abstract}
Objective: to compare standard color vision test results (Ishihara test) with a new test developed by the authors ("crayon" test) for the detection of congenital dyschromatopsia.

Methods: cross-sectional study of 712 children from three public schools and one private school in the city of Porto Alegre, state of Rio Grande do Sul. Children with learning disabilities, or systemic and ocular diseases were excluded from this random sample. Two color vision tests, Ishihara test (short version with 14 plates) and crayon test (developed by the authors) were sequentially applied. Each test was applied by different evaluators and analyzed by a third evaluator.

Results: the crayon test showed a specificity of $100 \%$ (99.3$100 \%)$ and sensitivity of $38.5 \%$ (15.1-67.7\%) when compared to Ishihara test. The prevalence of congenital dyschromatopsia in this population sample was $2.6 \%$ for male children, and $0.9 \%$ for female children.

Conclusions: the crayon test results showed greater specificity than Ishihara test in the studied group; however, sensitivity was not adequate for a screening test. It is necessary to improve the sensitivity so that congenital dyschromatopsia can be detected by the crayon test.
\end{abstract}

J Pediatr (Rio J) 2001; 77 (4): 327-30: color vision test, dyschromatopsia, Ishihara test, school children's health.

em crianças é um desafio. Quando pequenas, crianças afetadas pela discromatopsia congênita podem apresentar dificuldades com material educacional, sendo julgadas por professores e colegas como pouco inteligentes ou colaborativas. Portanto, o diagnóstico precoce minimiza tais problemas, melhora a adaptação das crianças à sua disfunção e auxilia seu planejamento profissional futuro ${ }^{2}$.

O diagnóstico da discromatopsia congênita é feito atualmente com testes cujo maior problema é seu alto custo e baixa aplicabilidade prática em grandes amostras populacionais. Por essas razões, os autores elaboraram o teste do giz de cera, que prima pelo seu baixo custo e fácil compreensão. 
Este trabalho tem por objetivo comparar os resultados obtidos na aplicação do teste de Ishihara (edição resumida com 14 pranchas) com os obtidos na aplicação do teste do giz de cera.

\section{Pacientes e Métodos}

Foi realizado um estudo transversal com 712 escolares (382 meninos e 330 meninas), escolhidos em diferentes pontos da Grande Porto Alegre, pertencentes à rede pública (3 escolas) e privada (1 escola) de Porto Alegre, Rio Grande do Sul, durante um período de 2 meses (1/10/99 a 30/11/ 99). A escolha das escolas foi por conveniência. A idade das crianças variou de 6 a 12 anos. Os escolares foram excluídos segundo os critérios respondidos pelo(a) professor(a): (1) aprendizado avaliado como anormal para a idade e (2) presença de doenças sistêmicas (retardamento mental, doenças metabólicas e diabetes juvenil) e oculares (glaucoma congênito, estrabismo e catarata congênita) reconhecidas. Não houve informação sobre qualquer dos escolares apresentar discromatopsia congênita (ou daltonismo). Os escolares foram examinados após autorização por escrito do(a) professor(a) e dos pais. O exame foi realizado individualmente na respectiva escola de cada criança. Os dois testes de visão de cores (teste de Ishihara- versão resumida com 14 pranchas e o teste do giz de cera, desenvolvido pelos autores) foram aplicados seqüencialmente.

Os alunos foram orientados em grupo sobre o preenchimento correto do teste do giz de cera. Esse teste consiste de 8 quadrados simétricos dispostos em duas linhas. Na primeira linha existem 4 quadrados pintados com giz de cera com as cores vermelho, verde, azul e amarelo em sequiência aleatória. As crianças eram solicitadas a pintar o quadrado de linha de baixo com a mesma cor do quadrado de cima (Figura 1). Para isso, foram colocados à disposição das crianças bastões de giz de cera em 12 cores diferentes dispostos aleatoriamente sobre uma mesa. O exame era considerado alterado se um quadrado de baixo fosse pintado com uma cor diferente do quadrado de cima. Após o teste do giz de cera, foi realizado o teste de Ishihara, versão resumida de 14 pranchas. O local de exame era iluminado com luz diurna, sendo que as pranchas pseudo-isocromáticas foram colocadas a uma distância de $75 \mathrm{~cm}$, formando um ângulo reto em relação à linha de visão. O teste era considerado alterado se o escolar cometesse sete ou mais erros na leitura das pranchas, conforme orientação do autor.

Cada teste (teste de Ishihara e teste do giz de cera) foi aplicado por um examinador diferente (M.B.M. e J.C.C.)* sob supervisão (M.F.B.)* e interpretado por um terceiro examinador (G.M.M.)*. Os examinadores foram treinados por preceptor do Setor de Oftalmologia Pediátrica (N.T.)*
Testes com resultados alterados foram repetidos e, quando confirmados, os pais ou responsáveis pela criança eram informados e orientados sobre a patologia pelos autores.

Este estudo foi aprovado pelo comitê de ética da Irmandade da Santa Casa de Misericórdia de Porto Alegre, RS.

\section{Resultados}

$\mathrm{O}$ teste do giz de cera e o teste de Ishihara foram realizados em 712 escolares, no período. A idade das crianças variou de 6 a 12 anos (média=8,5 anos). Destas, 26 crianças tinham 6 anos completos na data do exame, 190 tinham 7 anos, 183 tinham 8 anos, 125 tinham 9 anos, 101 tinham 10 anos, 70 tinham 11 anos e somente 17 tinham 12 anos. Neste grupo, $382(53,7 \%)$ eram meninos e $330(46,3 \%)$ eram meninas, $626(87,8 \%)$ brancos, $39(5,5 \%)$ negros e 47 $(6,7 \%)$ mestiços. Das crianças estudadas $5(0,7 \%)$ tiveram o teste do giz de cera alterado e $13(1,8 \%)$ mostraram alteração no teste de Ishihara. Todas as 5 crianças com o teste do giz de cera alterado eram meninos e, dentre as crianças com teste de Ishihara patológico, 10 eram meninos e 3 eram meninas.

Quando comparamos o teste do giz de cera com o teste de Ishihara, observamos que 5 pacientes foram positivos para ambos os testes. $\mathrm{O}$ teste do giz de cera apresenta uma sensibilidade de $38,5 \%(15,1-67,7 \%)$ e uma especificidade de $100 \%(99,3-100,0 \%)$. escolares estudados 
Na população de meninos, a prevalência de discromatopsia congênita detectada pelo teste de Ishihara foi de $2,6 \%(1,3-4,8 \%)$. A sensibilidade do teste do giz de cera em relação ao teste de Ishihara no grupo dos meninos foi de $50 \%(20,1-79,9 \%)$ e a especificidade de $100 \%$ (98,7$100,0)$. Dentro da população feminina, o teste do giz de cera não detectou pacientes com alterações. Entretanto, o teste de Ishihara estabeleceu a existência de 3 meninas com discromatopsia congênita (Tabela 1).

Em relação à prevalência da discromatopsia congênita nesta amostra, encontramos 2,6\% dos homens e 0,9\% das mulheres com a patologia. Esse achado está apresentado na Figura 2 e comparado com outros estudos de incidência da discromatopsia congênita.

\section{Discussão}

A causa mais comum de deficiência na visão das cores é a discromatopsia congênita. Essa é sempre bilateral, não modifica com o tempo e não tem tratamento ${ }^{1}$. Para estabelecer o diagnóstico de certeza, deve ser excluída doença do nervo óptico e/ou mácula no paciente afetado ${ }^{1}$. Existem autores que acreditam que retardamento mental e doenças oculares como glaucoma congênito, retinopatia diabética e ambliopia, ou até o uso de drogas antiepilépticas, possam causam alterações na visão de cores, ocasionando falsopositivos nos testes de rastreamento da discromatpsia congênita $^{1,2}$. Entretanto, estudos atuais revelaram que essas influências podem não ser verdadeiras ${ }^{3-5}$.

O diagnóstico da discromatopsia congênita pode ser feito através de vários testes como o Teste de Ishihara, o Farnsworth Munsell, o anomaloscópio de Nagel, entre outros $^{2,6}$. Através dos anos, vários estudiosos tentaram aperfeiçoá-los, mas, até hoje, nenhum teste é considerado completo 7 .
O teste das pranchas padronizadas de Ishihara, que é extremamente sensível para os defeitos verde-vermelho, não é adequado para os defeitos azul-amarelo ${ }^{8}$. É um teste quantitativo, originalmente elaborado para os casos de discromatopsias congênitas e, por isso, não possui nenhum efeito para as discromatopsias adquiridas, como a neurite óptica, retinose pigmentare coriorretinite. O teste de Ishihara é até hoje o mais utilizado no mundo todo para a detecção da discromatopsia congênita.

Em relação aos outros testes, o Farnsworth-Munsell 100-cores é um teste qualitativo, sendo também designado para os casos de discromatopsias congênitas. Permite o diagnóstico de qualquer tipo e grau de discromatopsia. É pouco utilizado na clínica prática, pois é demorado - em média 20 minutos para cada olho9 ${ }^{9}$ O anomaloscópio de Nagel é um aparelho utilizado pelo oftalmologista, dificultando seu uso em grandes amostras populacionais. No teste de Holmgren, o paciente deve retirar pedaços de lãs de

Figura 2 - Comparativo das prevalências da discromatopsia congênita obtidas neste estudo (RS) e em estudos publicados por Campos (RJ) e Swanson et al. (EUA)

Tabela 1 - Comparação entre o teste do giz de cera e o teste de Ishihara na amostra populacional estudada; os valores citados são a porcentagem observada na amostra seguido do intervalo de confiança entre parênteses

\begin{tabular}{lcll}
\hline & Meninos & Meninas & Geral \\
\hline $\begin{array}{l}\text { Sensibilidade (\%) } \\
\text { Especificidade (\%) }\end{array}$ & $50(20,1-79,9)$ & $0(0,0-70,8)$ & $38,5(15,1-67,7)$ \\
$\begin{array}{l}\text { Valor Preditivo } \\
\text { Positivo (\%) }\end{array}$ & $100(98,7-100)$ & $100(98,9-100)$ & $100(99,3-100)$ \\
$\begin{array}{l}\text { Valor Preditivo } \\
\text { Negativo (\%) }\end{array}$ & $98,7(96,8-99,5)$ & - & $100(46,3-100)$ \\
& & & $99,1(97,4-99,8)$ \\
\hline
\end{tabular}


diferentes cores para compará-las com as amostras escolhidas pelo examinador. A desvantagem deste teste é a alteração da tonalidade das amostras decorrente das manipulações freqüentes e da exposição prolongada à luz. Outros testes são a lanterna de Edrige-Green, as pranchas pseudoisocromáticas de Dvorine, o American Optical-Hardy-RandRittler (não disponível comercialmente) e o APT- $5^{6}$. Um novo teste de tabelas isocromáticas (color vision testing made easy - CVTME) foi criado com o objetivo de avaliar indivíduos de todas as idades ${ }^{10}$. O CVTME se mostrou $100 \%$ específico e $90,5 \%$ específico na detecção da discromatopsia congênita ${ }^{10}$. Entretanto, seu custo alto e disponibilidade ainda limitada, impossibilitaram sua comparação com o teste aqui apresentado.

Observa-se que os testes para a detecção da discromatopsia congênita continuam sendo aprimorados até o presente $^{9,10}$. Dessa forma, tais testes tornam-se caros e complexos para a aplicação em grandes grupos populacionais, principalmente em países pobres. Por essas razões, os autores se propuseram a criar um teste simples, barato, de fácil aplicação e interpretação. Para avaliar sua validade, comparamos seus resultados com um teste altamente sensível para deficiências vermelho-verde, que é o teste de Ishihara. Apesar da alta especificidade do teste do giz de cera, sua sensibilidade é baixa em relação ao teste de Ishihara, o que o torna impróprio para rastreamento. Entretanto, pode ser aplicado num primeiro momento por médicos pediatras ou professores primários, quando a família tiver dúvidas quanto à capacidade da criança em diferenciar as cores. Devido a sua alta especificidade, uma vez que o resultado seja anormal, a criança testada certamente terá a discromatopsia congênita. Caso o resultado seja normal, a criança terá ainda $1,1 \%$ de chances de ser portador da patologia.

$\mathrm{Na}$ amostra estudada, encontramos um número inferior de homens afetados pela discromatopsia congênita que estudos prévios realizado no Rio de Janeiro ${ }^{8}$ e em outros países ${ }^{6}$. Entretanto, o número de mulheres afetadas pela discromatopsia congênita foi superior. Essas variações podem ser devidas a vários fatores, dentre eles a região estudada, possivelmente em virtude dos fatores genéticos da população. Além disso, a técnica utilizada na aplicação dos testes, bem como o fato desta amostra não ser representativa da população e ser pequena em comparação a outros estudos deste tipo podem ter influenciado os resultados.
Concluindo, o teste do giz de cera apresentou especificidade superior ao teste de Ishihara. Entretanto, sua sensibilidade é baixa em relação ao teste de Ishihara, tornandoo impróprio para rastreamento, devendo assim ser aperfeiçoado para ser usado com esta finalidade.

\section{Referências bibliográficas}

1. Stone E. Pediatric retinal disease. In: Wright KW, ed. Ophthalmology and strabismus. Philadelphia, London: Mosby; 1995. p431-580.

2. Pokorny J, Smith VC, Verriest G, Pinckers AJLG. Congenital and acquired color vision defects. New York, NY: Grune \& Stratton; 1979.

3. Erickson GB, Block SS. Testability of a color vision screening test in a population with mental retardation. J Am Optom Assoc 1999; 70: 758-63.

4. Lopez L, Thomson A, Rabinowicz Al. Assessment of color vision in epileptic patients exposed to single-drug therapy. Eur Neurol 1999; 41: 201-5.

5. Kocak-Altintas AG, Santana B, Kocak I, Duman S. Visual acuity and color deficiency in amblyopia. Eur J Ophthalmol 2000; 10:77-81.

6. Swanson WH, Everett M. Color vision screening of young children. J Pediatr Ophthalmol Strabismus 1992; 29:49-54.

7. Alves AA, Alves LFA. Discromatopsias. In: Alves AA, ed. Refração. 2a ed. Rio de Janeiro: Editora Cultura Médica; 1994. p 345-57.

8. Campos E. O daltonismo. Rev Bras Oftal 1949; 8:3-27.

9. Kon $\mathrm{CH}$, DeAlwis D. A new colour vision test for clinical use. Eye 1996; 10:65-74.

10. Cotter SA, Lee DY, French AL. Evaluation of a new color vision test: "color vision testing made easy". Optom Vis Sci 1999; 76: 631-6.

Endereço para correspondência:

Dra. Zélia M.S. Corrêa

Av. Nilo Peçanha, 2421

CEP 91330-001 - Porto Alegre, RS

Tel/Fax: (51) 3328.5164 - E-mail: zmcorrea@zaz.com.br 